\title{
Identification of Nanoparticle Prototypes and
}

\section{Archetypes: Supplementary Information}

\author{
Michael Fernandez* and Amanda S. Barnard \\ CSIRO Virtual Nanoscience Laboratory, 343 Royal Parade, Parkville 3052, Victoria, \\ Australia \\ E-mail: michael.fernandezllamosa@csiro.au
}

\section{Characteristics of the diamond nanoparticle datasets}

Our realistic ensemble of virtual nanoparticle samples contains considerable variety of shapes and a large range of sizes that cover the ranges observed experimentally and includes a total of 12 morphologies, as described in Table 1, and illustrated in Figure 1. In addition to this, based on the enthalpy of formation for each relaxed structure, we include the probability of observation within the data analytics (described below, and in the main text), calculated with a Boltzmann distribution at the formation temperature of $4500 \mathrm{~K}^{1}$

\footnotetext{
${ }^{*}$ To whom correspondence should be addressed

${ }^{1}$ Mochalin, V. N.; Shenderova, O.; Ho, D.; Gogotsi, Y. The Properties and Applications of Nanodiamonds. Nat. Nanotechnol. 2012, 7, 11-23.
} 
Table 1: Identifiers for geometric shapes included in this study, with different fractions of $\{100\},\{110\}$ and $\{111\}$ facets and their ratios in the nanodiamonds and bucky-diamond datasets.

\begin{tabular}{l|ccc|cc|c}
\hline Shape & $\begin{array}{c}\{11\}\} \\
(\%)\end{array}$ & $\begin{array}{c}\{110\} \\
(\%)\end{array}$ & $\begin{array}{c}\{100\} \\
(\%)\end{array}$ & $\begin{array}{c}\text { Nanodiamond } \\
\text { Ratio }\end{array}$ & $\begin{array}{c}\text { Bucky- } \\
\text { diamond } \\
\text { Ratio }\end{array}$ & Figure \\
\hline Regular hexahedron (Cube) & 0 & 0 & 100 & 3.30 & 5.13 & $1 \mathrm{a}$ \\
Truncated hexahedron & 11 & 0 & 89 & 3.85 & 6.84 & $1 \mathrm{~b}$ \\
Cuboctahedron & 37 & 0 & 63 & 10.99 & 6.84 & $1 \mathrm{c}$ \\
Truncated octahedron & 78 & 0 & 22 & 14.29 & 8.55 & $1 \mathrm{~d}$ \\
Regular octahedron & 100 & 0 & 0 & 4.95 & 7.69 & $1 \mathrm{e}$ \\
Rhombic dodecahedron & 0 & 100 & 0 & 4.95 & 7.69 & $1 \mathrm{f}$ \\
Doubly-truncated octahedron & 45 & 24 & 30 & 15.38 & 11.11 & $1 \mathrm{~g}$ \\
Small rhombicuboctahedron & 16 & 56 & 28 & 14.29 & 11.11 & $1 \mathrm{~h}$ \\
Great rhombicuboctahedron & 34 & 19 & 47 & 5.50 & 8.55 & $1 \mathrm{i}$ \\
Rhombi-hexahedron & 0 & 85 & 15 & 9.89 & 7.69 & $1 \mathrm{j}$ \\
Rhombi-octahedron & 24 & 76 & 0 & 7.69 & 11.11 & $1 \mathrm{k}$ \\
Doubly-truncated dodecahedron & 5 & 86 & 9 & 4.95 & 7.69 & $1 \mathrm{l}$ \\
\hline
\end{tabular}

(a)

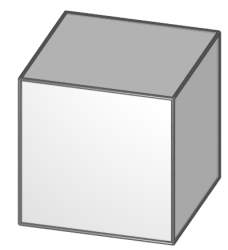

(b)

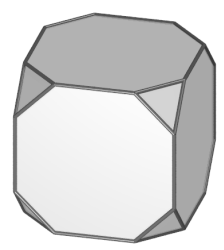

(c)

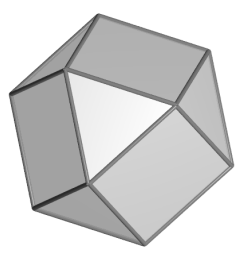

(d)

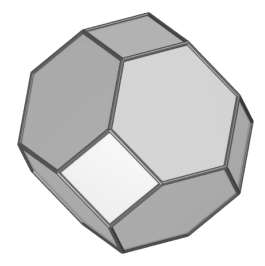

(g)

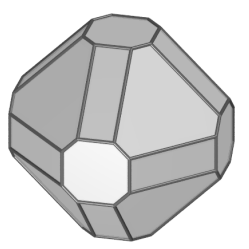

(f)

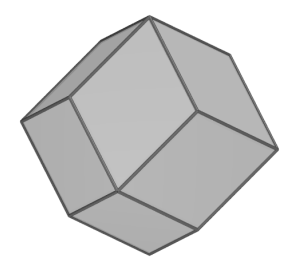

(e)
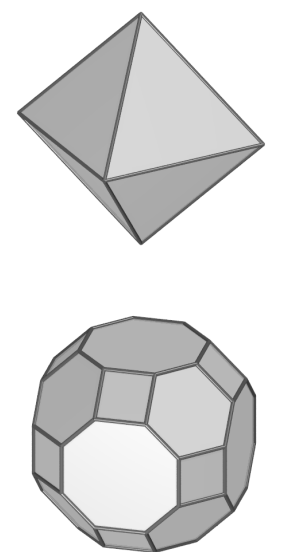

(i)

(j)

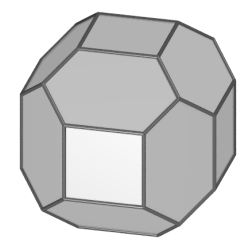

(k)

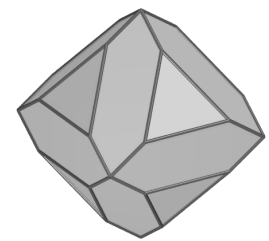

(h)

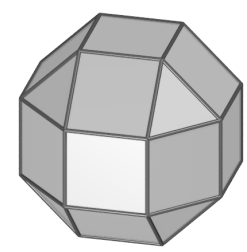

(1)

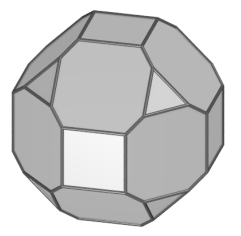

Figure 1: Schematic representations of the nanoparticle morphologies included in this study: (a) regular hexahedron (cube), (b) truncated hexahedron, (c) cuboctahedron (d) truncated octahedron, (e) regular octahedron, (f) rhombic dodecahedron, (g) doubly-truncated octahedron, (h) small rhombicuboctahedron, (i) great rhombicuboctahedron, (j) rhombi-hexahedron, (k) rhombi-octahedron, (l) doubly-truncated dodecahedron. 


\section{Principal Component Analysis (PCA), $k$-means clustering and archetype analysis (AA)}

\section{Selection of principal components, clusters and archetypes}

The selection of the optimum number of principal components (PC), clusters and archetypes was performed by analysing the amount of explained data variance as a function of the number of components in each analysis in Figure 2 and Figure 3. As we can observed, $\sim 87 \%, \sim 65 \%$ and $\sim 87 \%$ of the data variances of the nanodiamonds was described by the

first three PCs, five clusters and five archetypes, respectively. In the case of the buckydiamonds, the first three PCs, six clusters and seven archetypes describe $\sim 66 \%, \sim 65 \%$ and $\sim 71 \%$ of data variance, respectively. Meanwhile for the passivated graphenes, the first three PCs, seven clusters and five archetypes describe $\sim 80 \%, \sim 73 \%$ and $\sim 88 \%$ of data variance. In the case of the unpassivated graphenes, the first three PCs, seven clusters and five archetypes describe $\sim 92 \%, \sim 79 \%$ and $\sim 95 \%$ of data variance. 

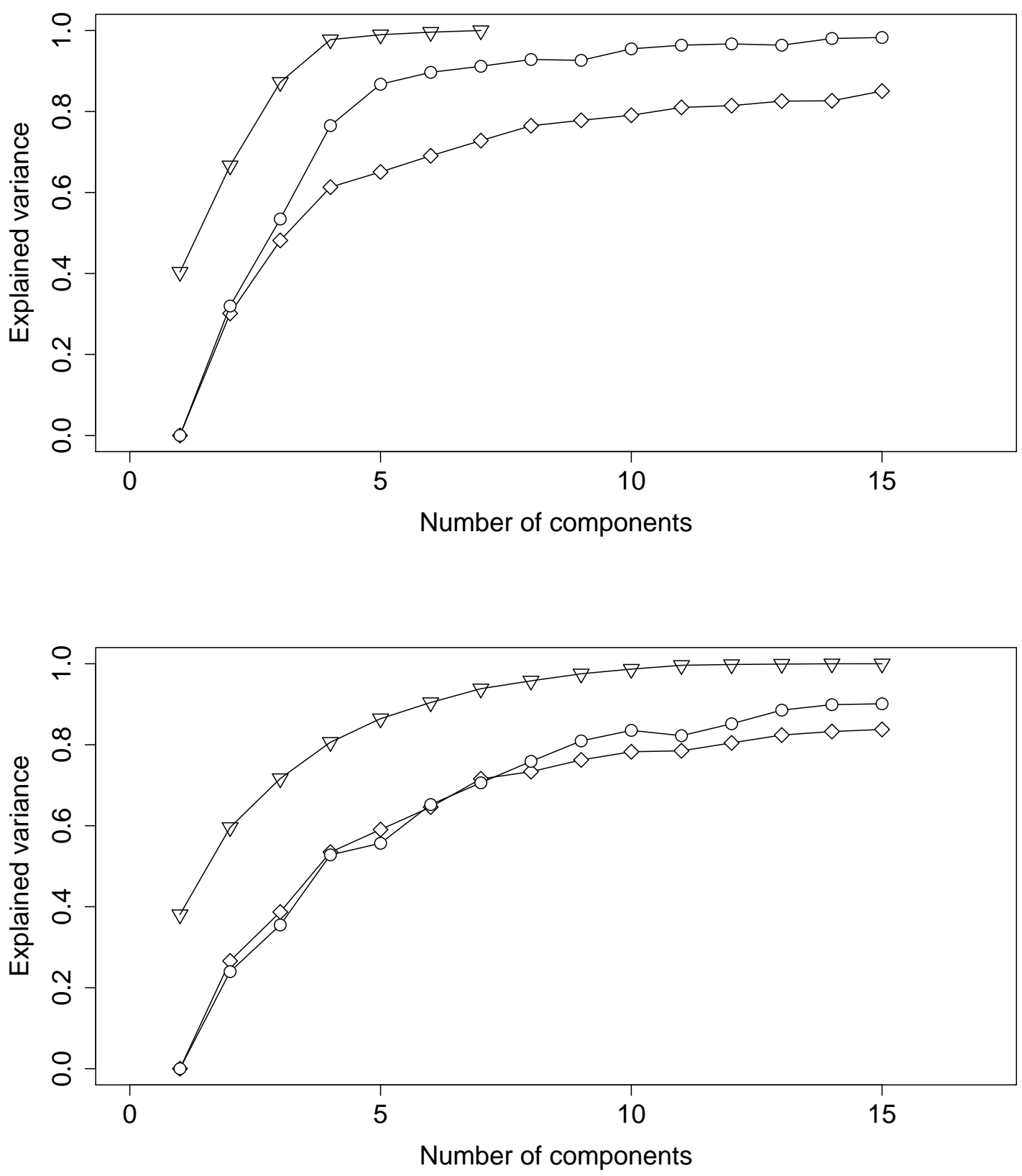

Figure 2: Amount of described variance of the (a) nanodiamonds and (b) bucky-diamonds by the PCA $(\nabla), k$-means $(\diamond)$ and $\mathrm{AA}(\circ)$. 

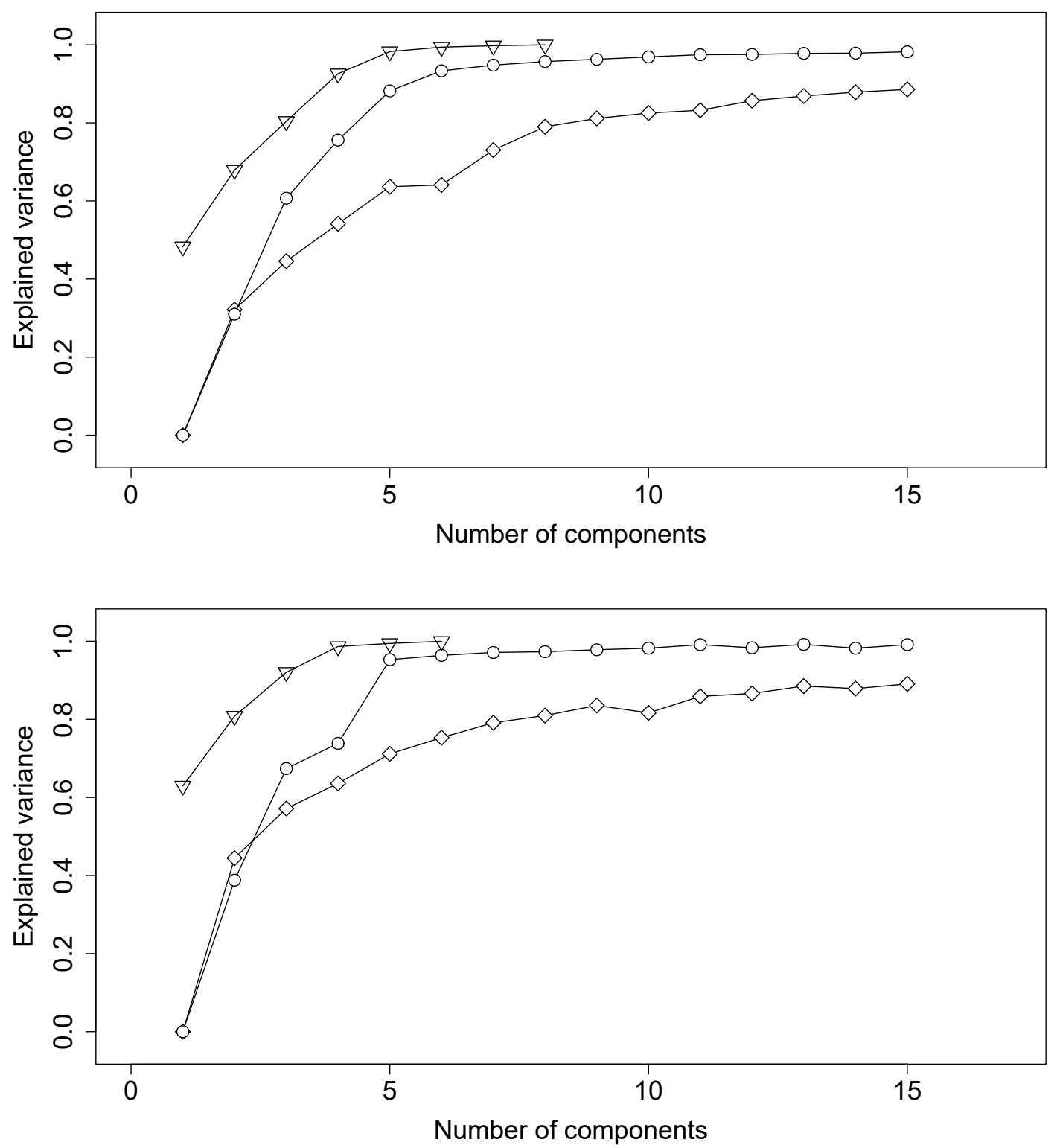

Figure 3: Amount of described variance of the (a) passivated graphenes and (b) unpassivated graphenes by the PCA $(\nabla), k$-means $(\diamond)$ and $\mathrm{AA}(\circ)$.

\section{Contribution of the geometrical features to the principal components} (PCs)

The contributions of each geometrical features to the two main PCs of the nanodiamonds, bucky-diamonds, passivated graphenes and unpassivated graphenes appear in Figure 4, Fig- 
ure 5, Figure 6 and Figure 7, respectively.
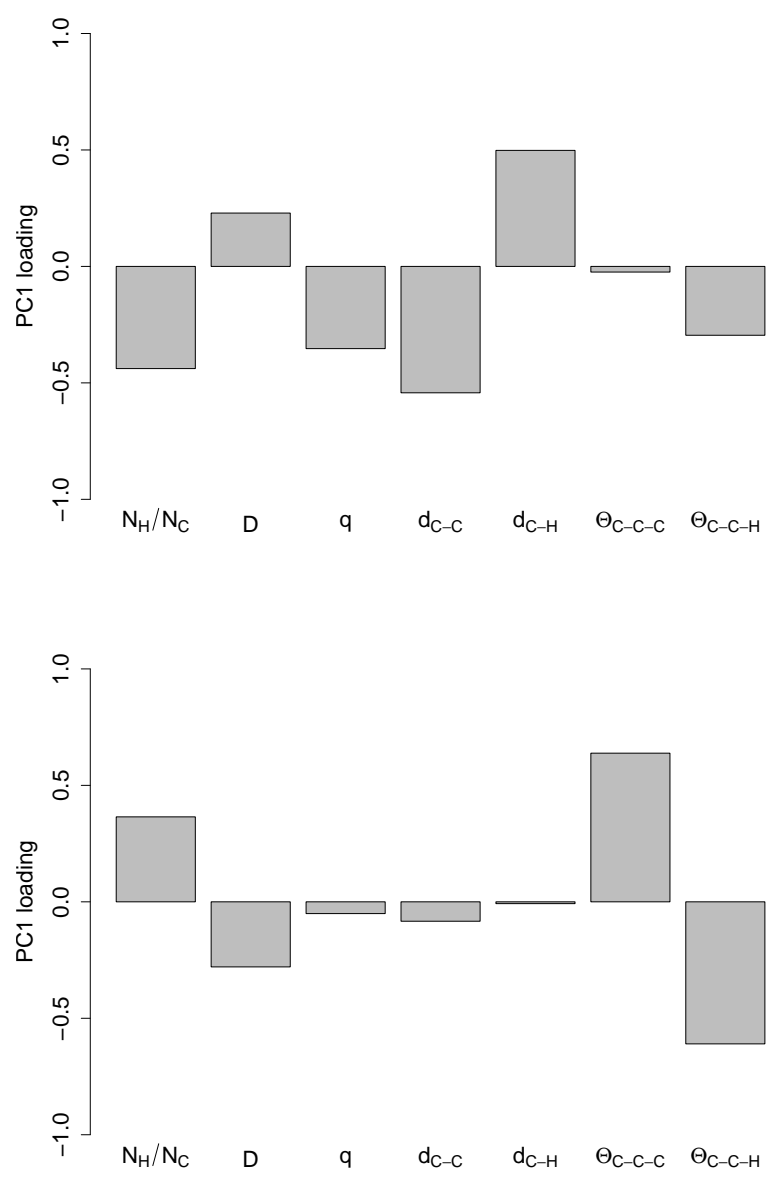

Figure 4: Loading of each geometrical features in the (a) PC1 and (b) PC2 of the nanodiamond dataset. 

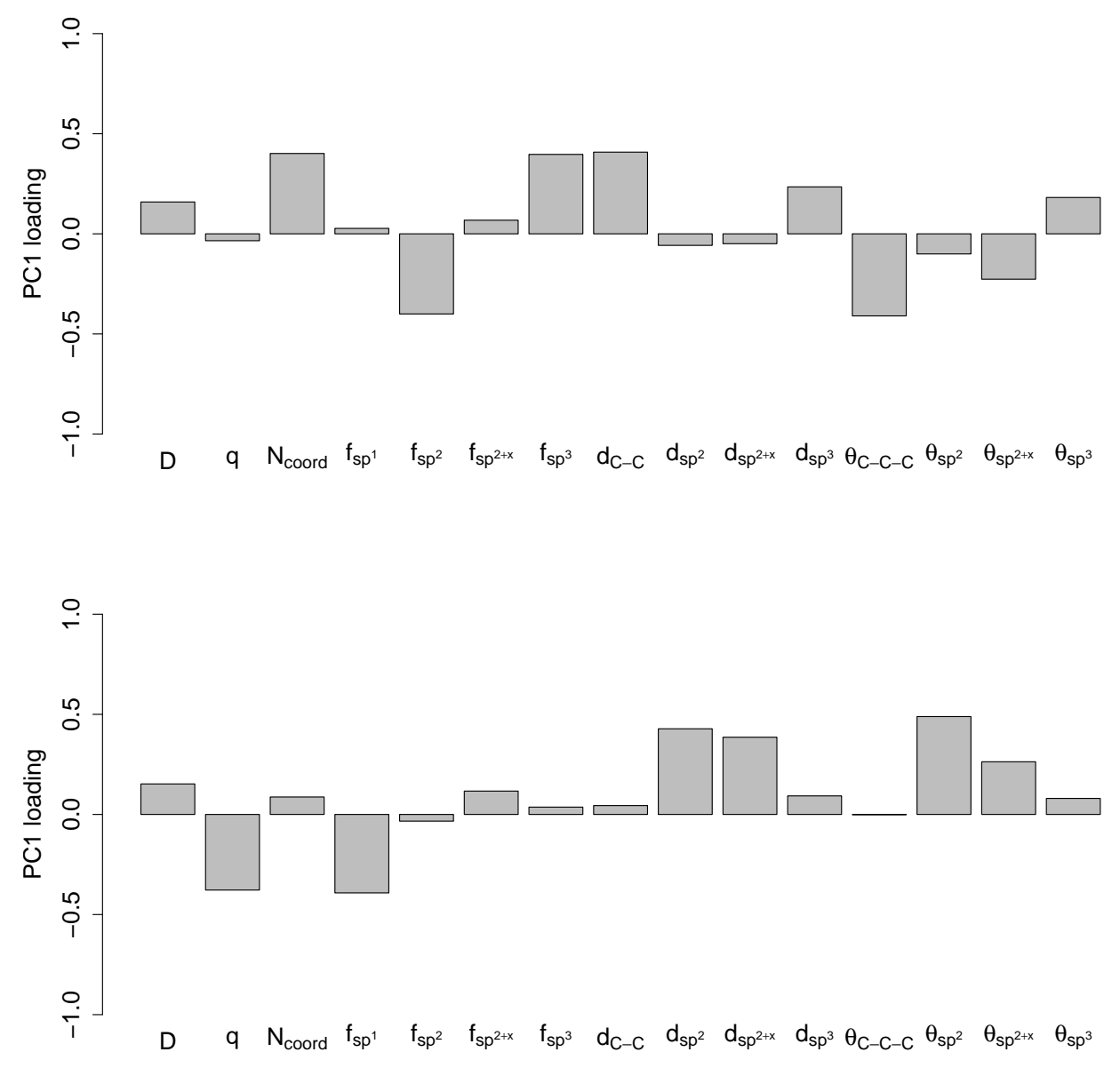

Figure 5: Loading of each geometrical feature in the (a) PC1 and (b) PC2 of the buckydiamond dataset. 

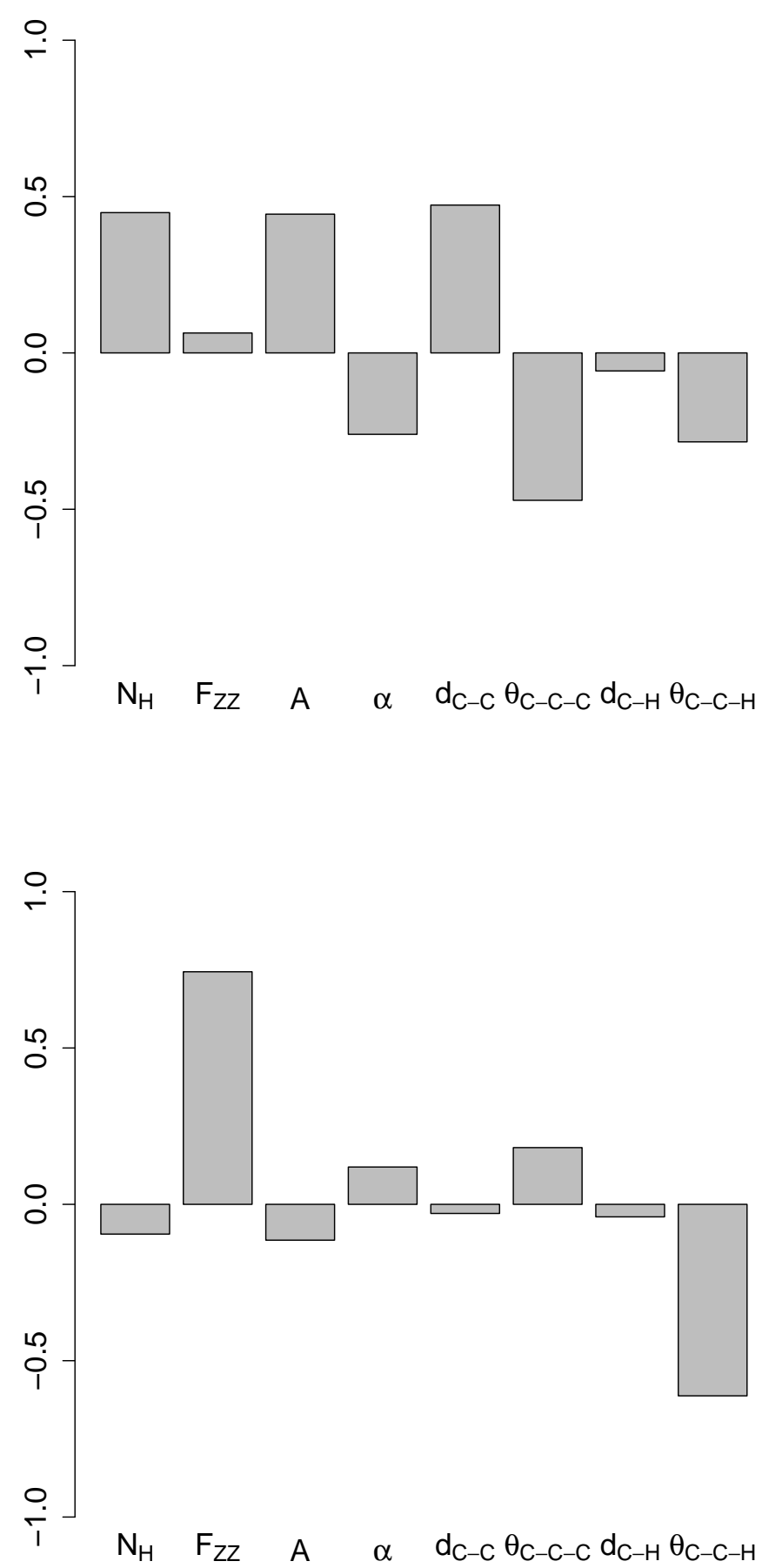

Figure 6: Loading of each geometrical features in the (a) PC1 and (b) PC2 of the passivated graphene dataset. 

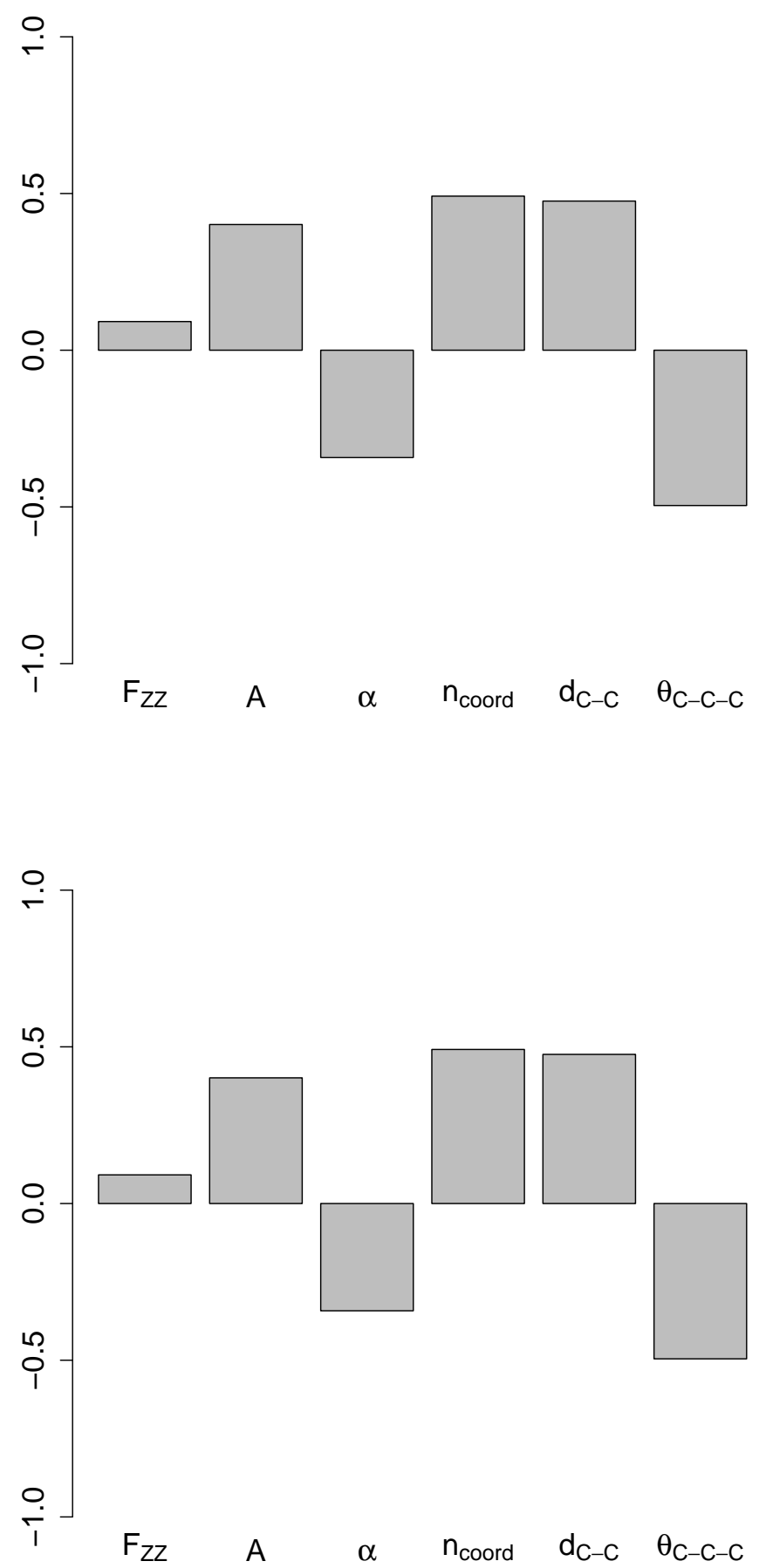

Figure 7: Loading of each geometrical feature in the (a) PC1 and (b) PC2 of the unpassivated graphene dataset. 


\section{Hierarchical clustering of the nanoparticle prototypes and archetypes}

To illustrate the diversity of the nanoparticles groups or clusters, we performed a hierarchical clustering of the prototype and archetype nanostructures by measuring the similarity between their geometrical features at different levels. The resulting dendrograms appear in Figure 2 in the manuscript and Figures 8 and 9 in the Supplementary Information.

\section{Individual contributions of the archetypes to each diamond nanopar- ticle samples}

In the AA analysis each nanostructure sample in the dataset is described as a linear combinations of the archetypes according to the following equation, $X_{i}=\sum_{j=1}^{k} \alpha_{i j} Z_{j}$. The analysis of the $\alpha_{i j}$ values reveals the individual contribution of each archetype to the explained variance of each sample, which is depicted in the heatmap plots in Figure 8 and Figure 9. In the manuscript, the contribution of each archetype to a particular nanostructure is considered significant when the relative $\alpha_{i j}$ value of archetype $j$ for the nanostructure particle sample $i$ is higher than 0.5 .

\section{Explained Sample Variance (ESV) of the archetype analysis}

As a quality measure the deviation between the $n^{\text {th }}$ original data point $x_{n}$ and the derived

data point $\sum_{j=1}^{k} \alpha_{n j} Z_{j}$ based on the archetypes was calculated. The measure is given as the Explained Sample Variance (ESV) ranging between 0 and 1 where 1 is a perfect match.

$$
E S V_{i}=\frac{\left\|x_{n}\right\|^{2}-\left\|x_{n}-\sum_{j=1}^{k} \alpha_{n j} Z_{j}\right\|^{2}}{\left\|x_{n}\right\|^{2}}
$$

By evaluating these $E S V$ values, it is possible to state which data points are well described by the model. No conclusions should be made for data points where $E S V$ is low, because these data points are poorly described by the model. 

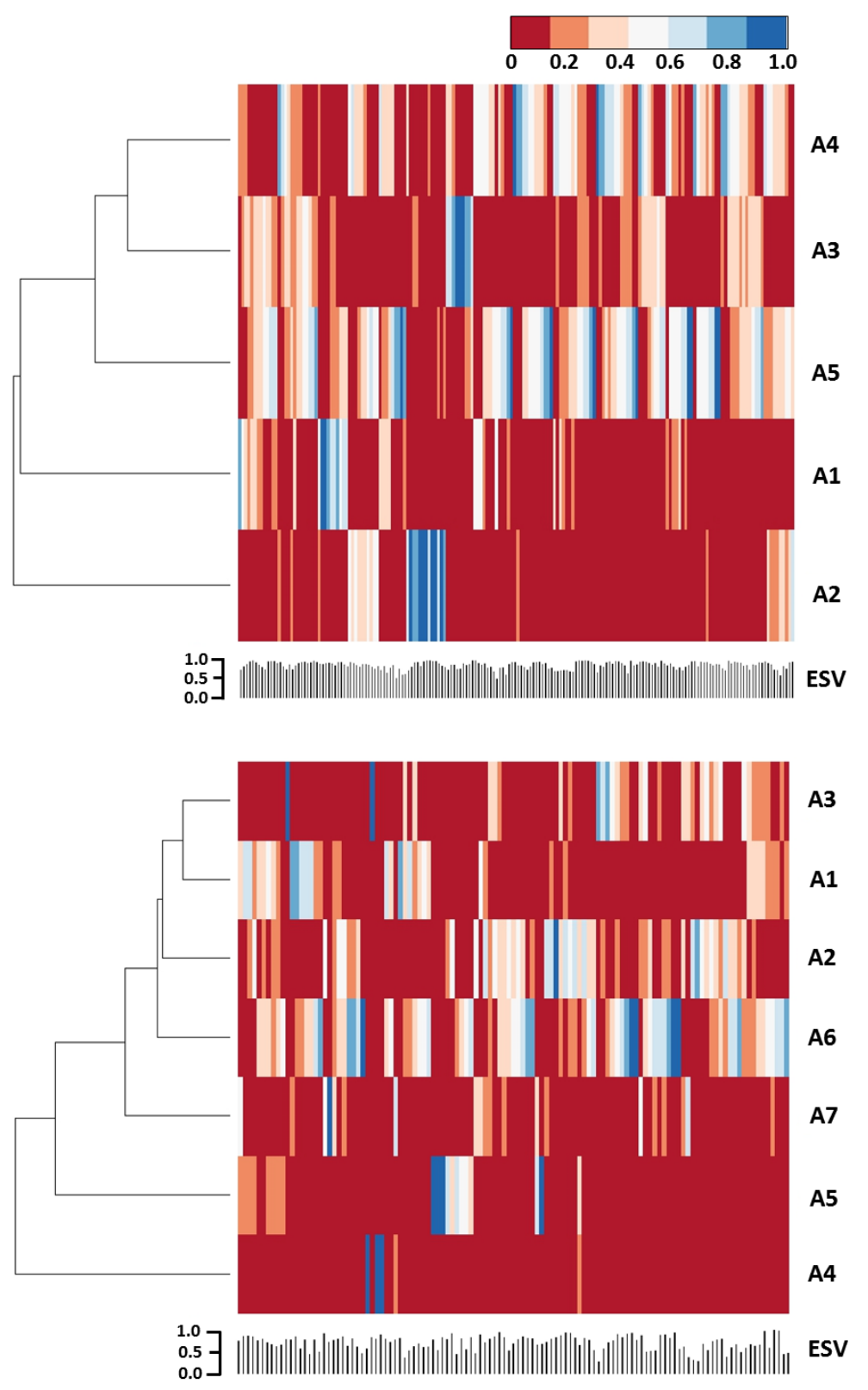

Figure 8: AA results depicted as the relation of (a) each nanodiamond and (b) each buckydiamond to the archetypes, shown as a heat map of the archetype coefficients. Each row represents one of the archetypes and each column represents a sample, and the colour indicates the degree to which the individual archetypes contribute to each sample. The values of explained sample variance $(E S V)$ are included to show how well the samples are described by the models. 

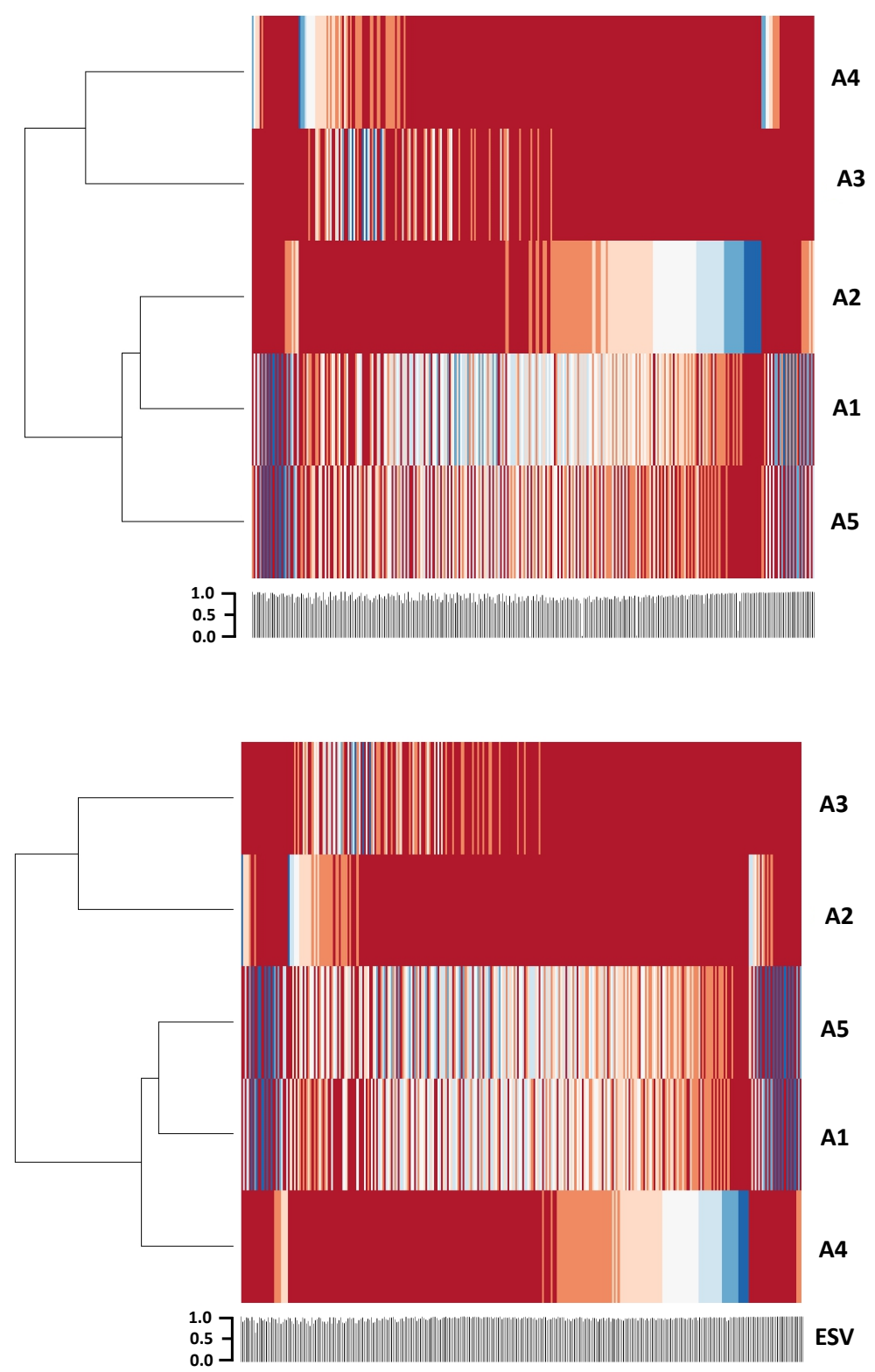

Figure 9: AA results depicted as the relation of (a) passivated graphene and (b) unpassivated graphene to the archetypes, shown as a heat map of the archetype coefficients. Each row represents one of the archetypes and each column represents a sample, and the colour indicates the degree to which the individual archetypes contribute to each sample. The values of explained sample variance $(E S V)$ are included to show how well the samples are described by the models. 


\title{
tutorial.r
}

\author{
mllamosa
}

Mon Nov 16 22:25:20 2015

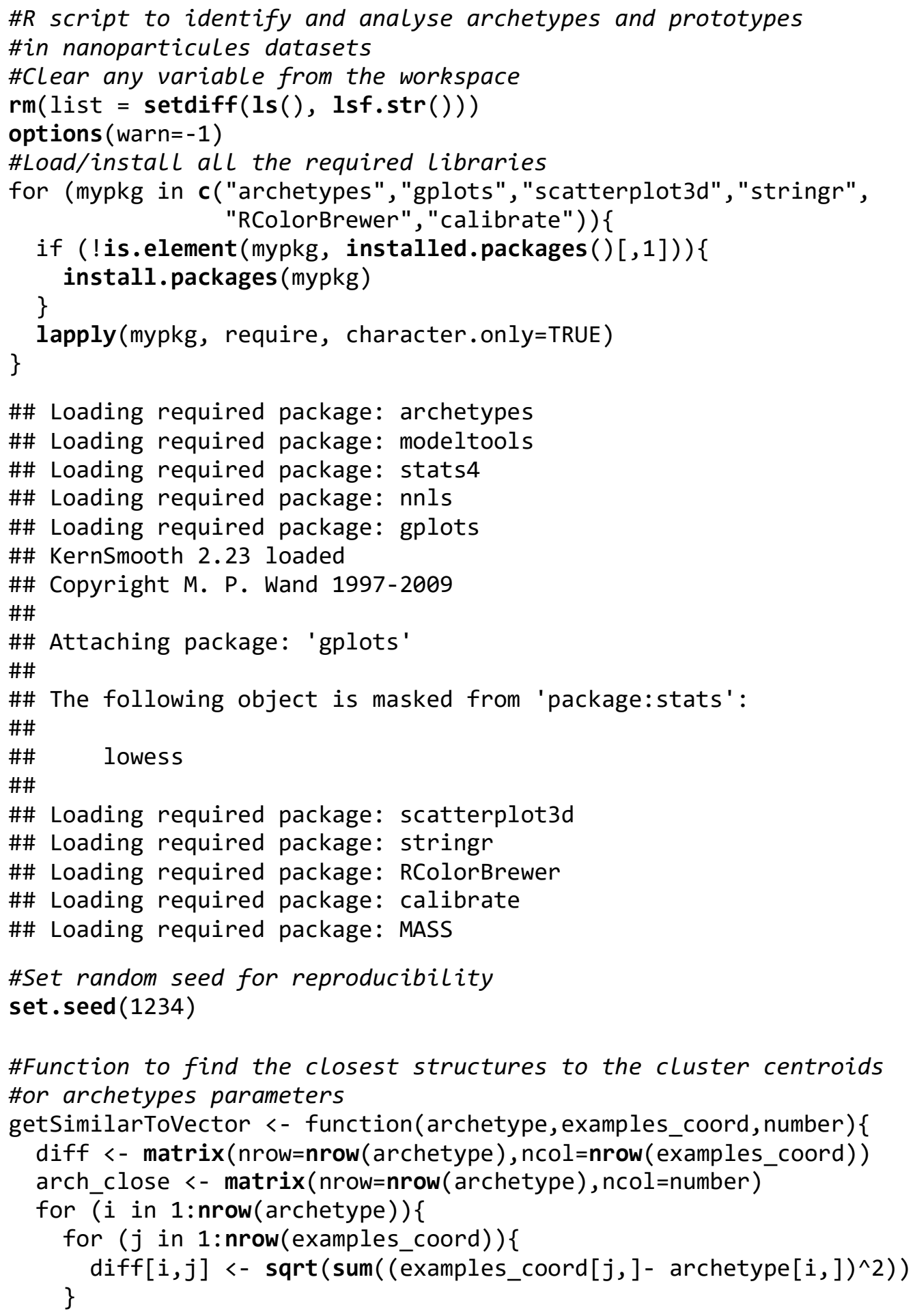




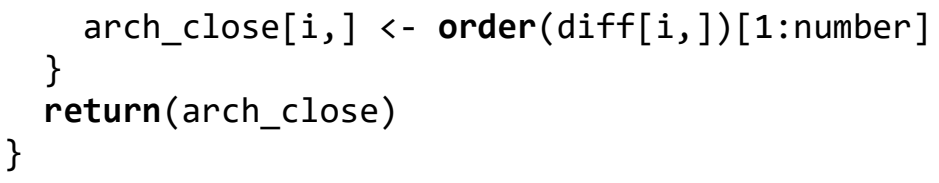




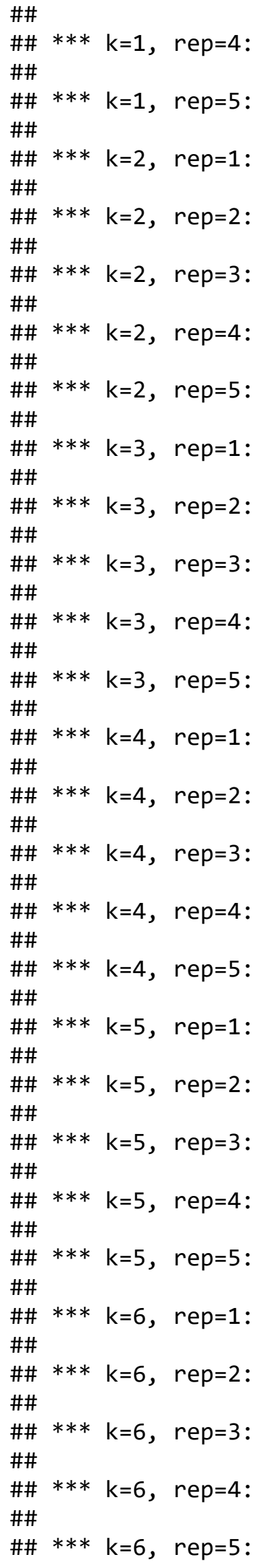




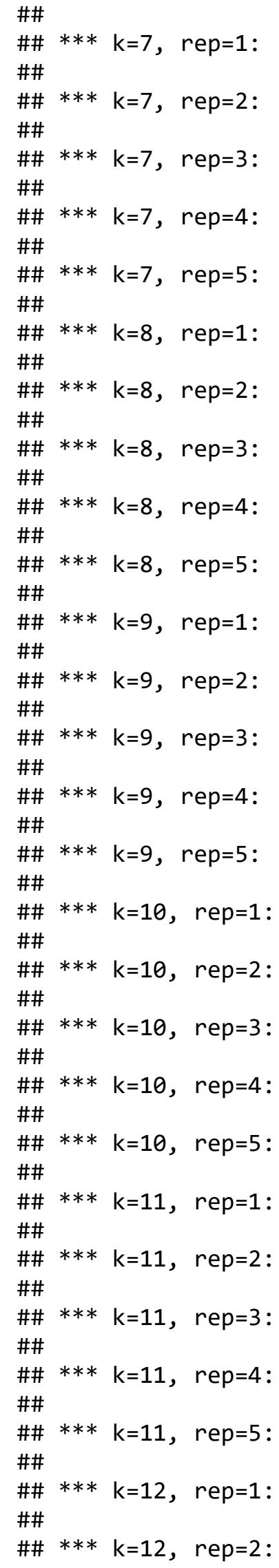




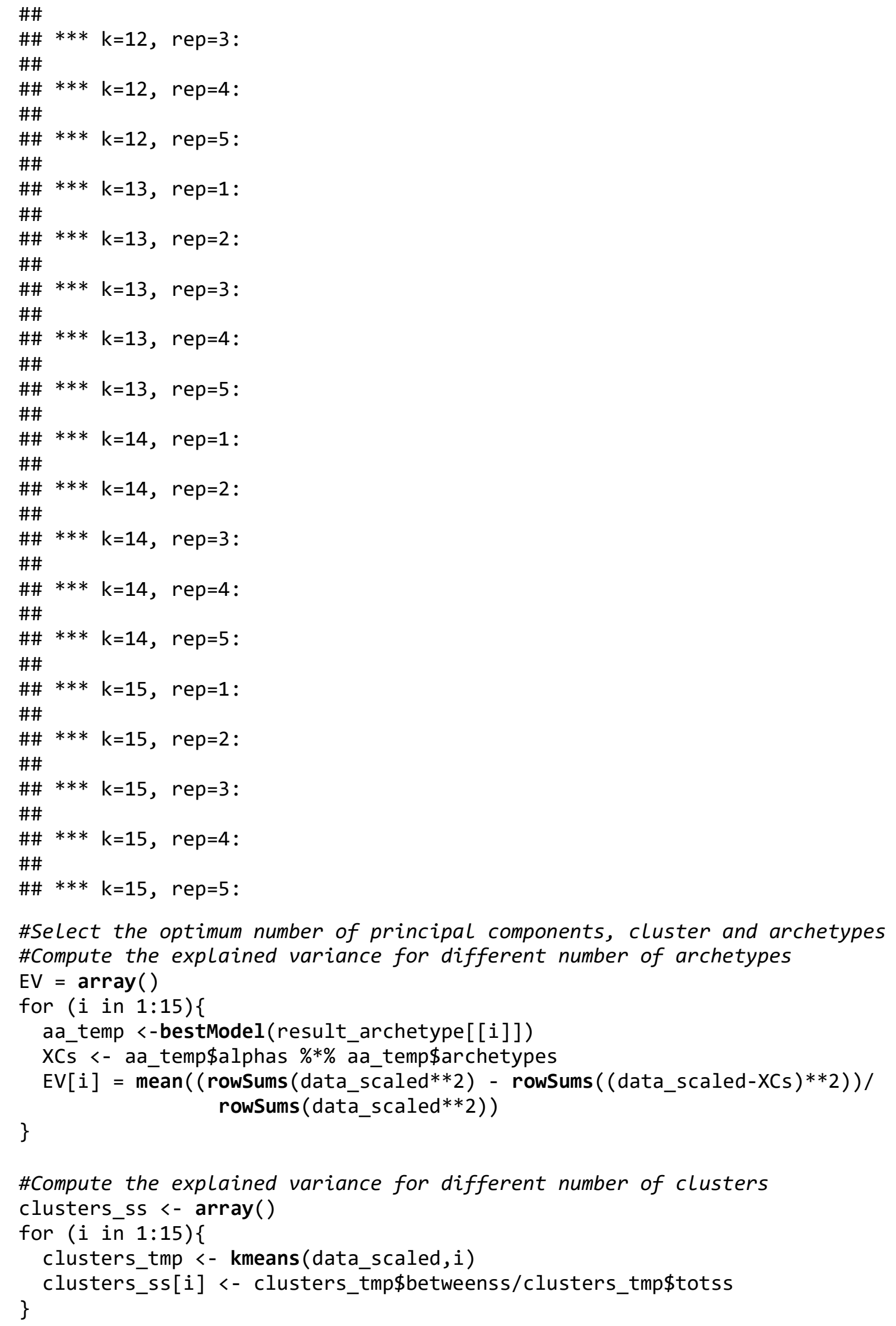


\#PLot the amount of explain variance vs. number of components

plot $($ clusters_ss, $y \lim =c(-0.040,1.04), x \lim =c(0,17), \mathrm{cex}=0.8, \mathrm{cex}$. axis $=$ 1.2 ,

$\mathrm{fg}=$ "black", $c o l=$ "white", $x l a b=$ "Number of components", ylab="Explained variance", cex.lab $=1.2$, pin=c $(7,5))$

lines(clusters_ss, col="black")

points(clusters_ss,pch $=23$, cex $=1.6$, fg = "black",bg = "white")

lines (EV, col="black")

points (EV,pch=21, cex = $1.6, \mathrm{fg}=$ "black",bg = "white")

points $(p c, p c h=25, c e x=1.6, f g=$ "black",bg = "white")

lines ( $p c, c o l=" b l a c k ")$

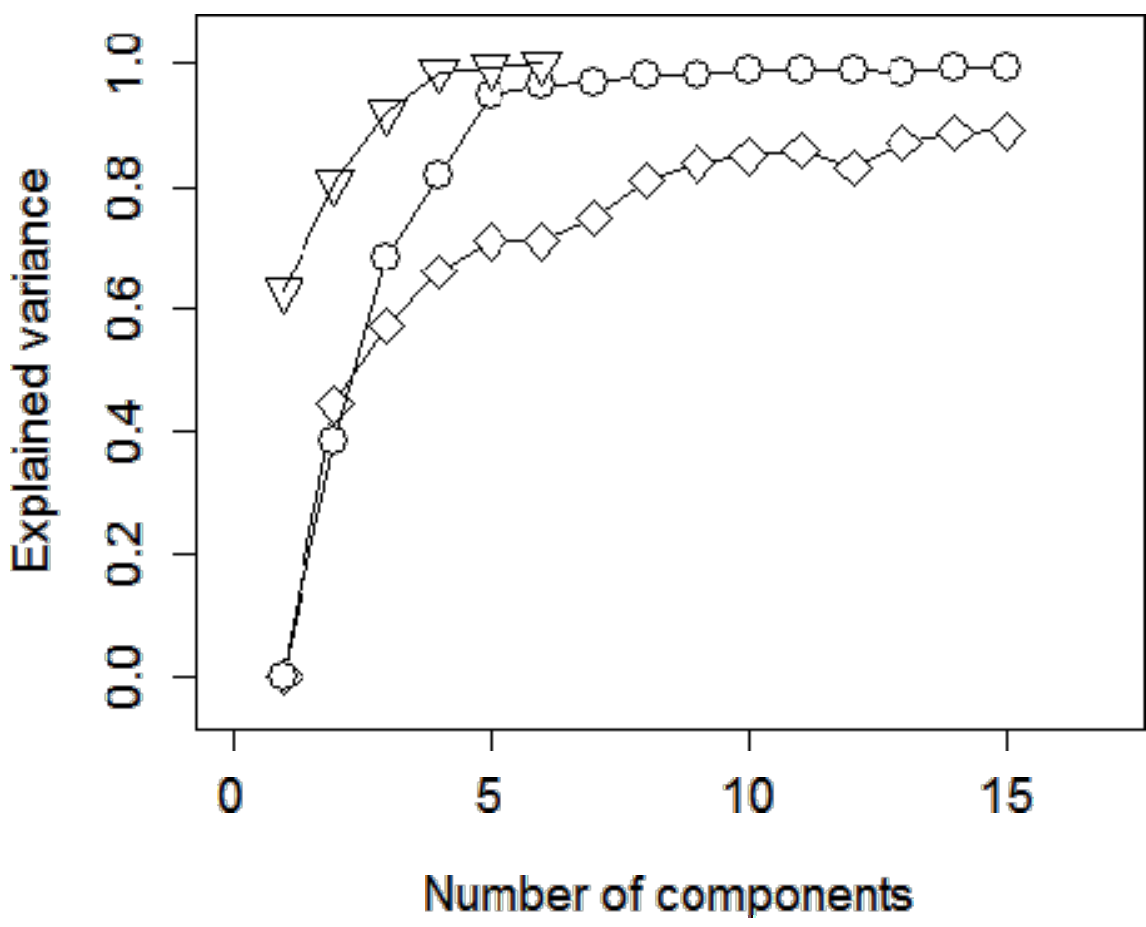

\#Set the optimum number of clusters and archetypes according to the \#elbow criteria in the plots

number_cluster $<-5$

number_archetype $<-5$

\#Compute the archetype model

aa <- bestModel(result_archetype[[number_archetype]])

\#PLot the dataset as simplex plots of the archetypes

pointcolor <- datasource[,color_ID] \#Coloring the points according a column value

\#Compute the sample explained variance

XCs <- aa\$alphas \%*\% aa\$archetypes

ESV $=$ (rowSums (data_scaled**2) - rowSums ( (data_scaled-

XCs) $* * 2)$ )/rowSums (data_scaled**2)

barplot $(E S V, y \lim =c(0,1.1)$, space $=10)$ 


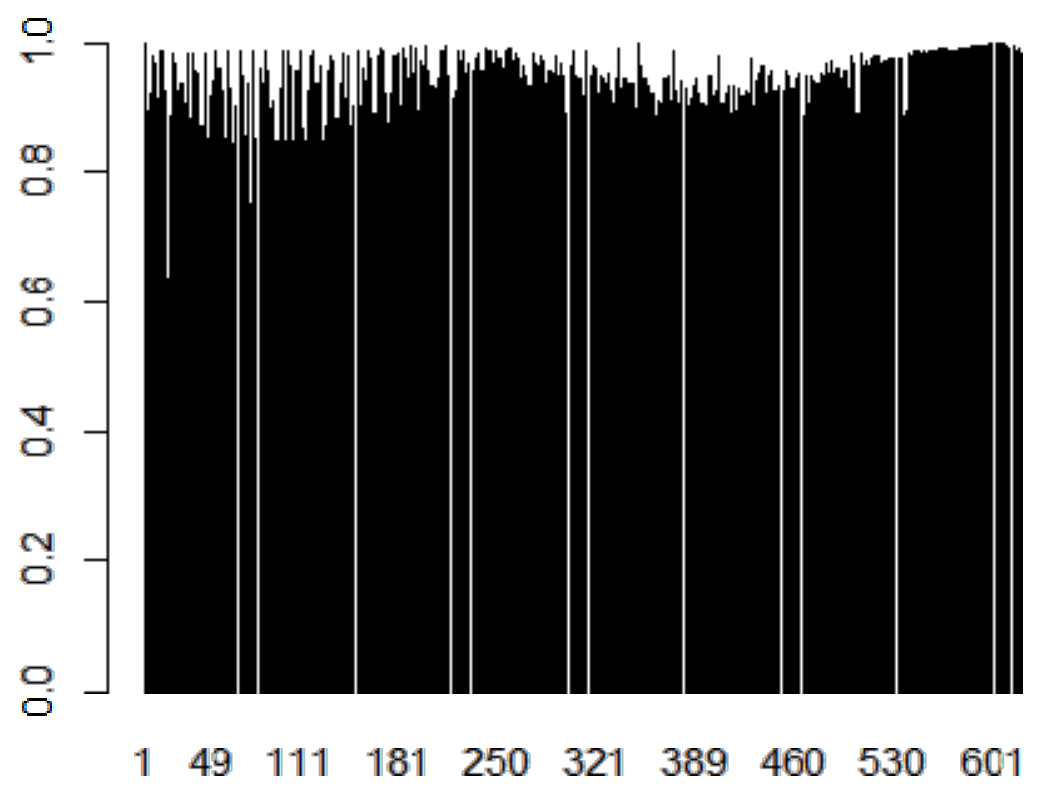

\#Compute the projection of the data in a simpleplot simplexplot (aa, points_col=pointcolor)

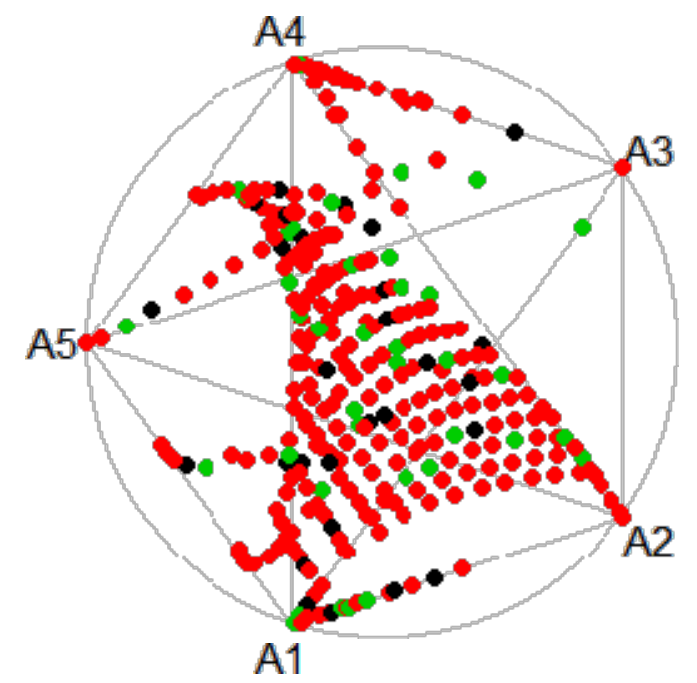

sp <- simplex_projection(aa\$archetypes)

\#Find the closest structure to the simplex nodes (archetypes) archetypal_Structures <- getSimilarToVector (sp, aa\$alphas\%*\%sp, 1) 
\#Compute the clusters

clusters <- kmeans(data_scaled, number_cluster)

\#Find the closest structure to the cluster centroids (prototypes)

clusters_Structures <- getSimilarToVector(clusters\$centers, data_scaled,1)

\#Build a dendrogram to compare the archetypes

hc_structure <- hclust(dist(data_scaled[archetypal_structures, ]))

plot(hc_structure)

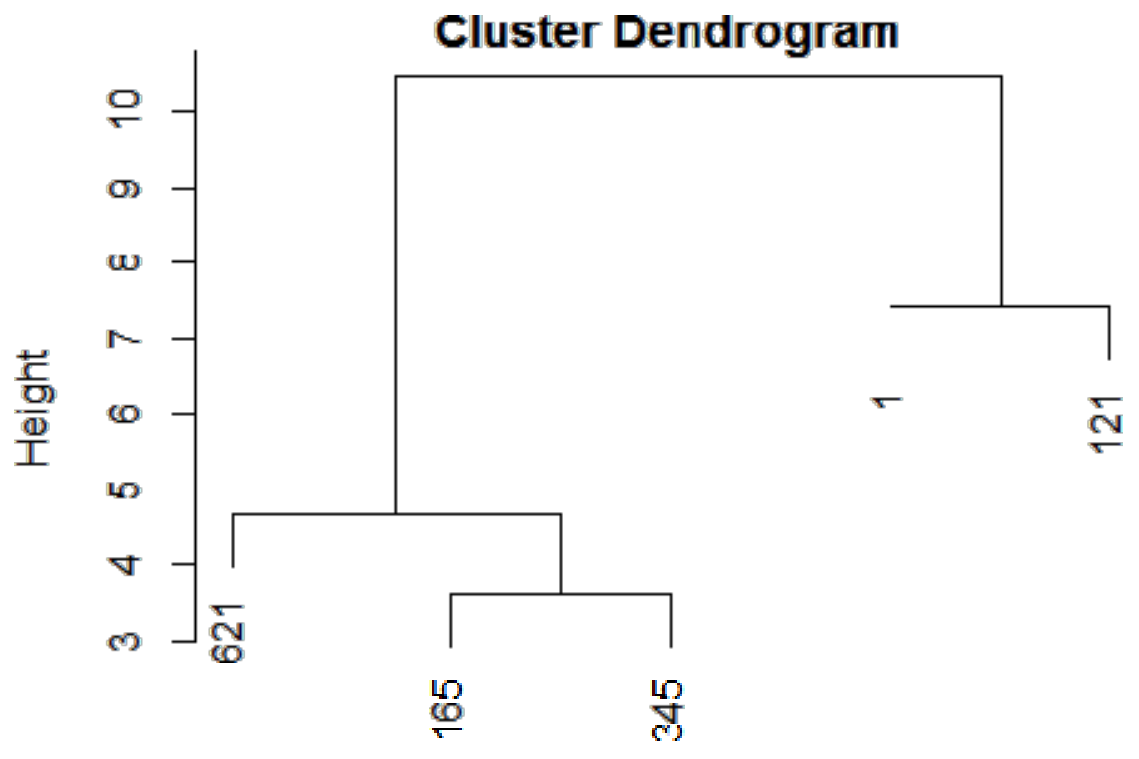

dist(data_scaled[archetypal_Structures, ])

\#Build a dendrogram to compare the prototypes

hc_structure_clusters <- hclust(dist(data_scaled[clusters_Structures, ])) plot(hc_structure_clusters) 


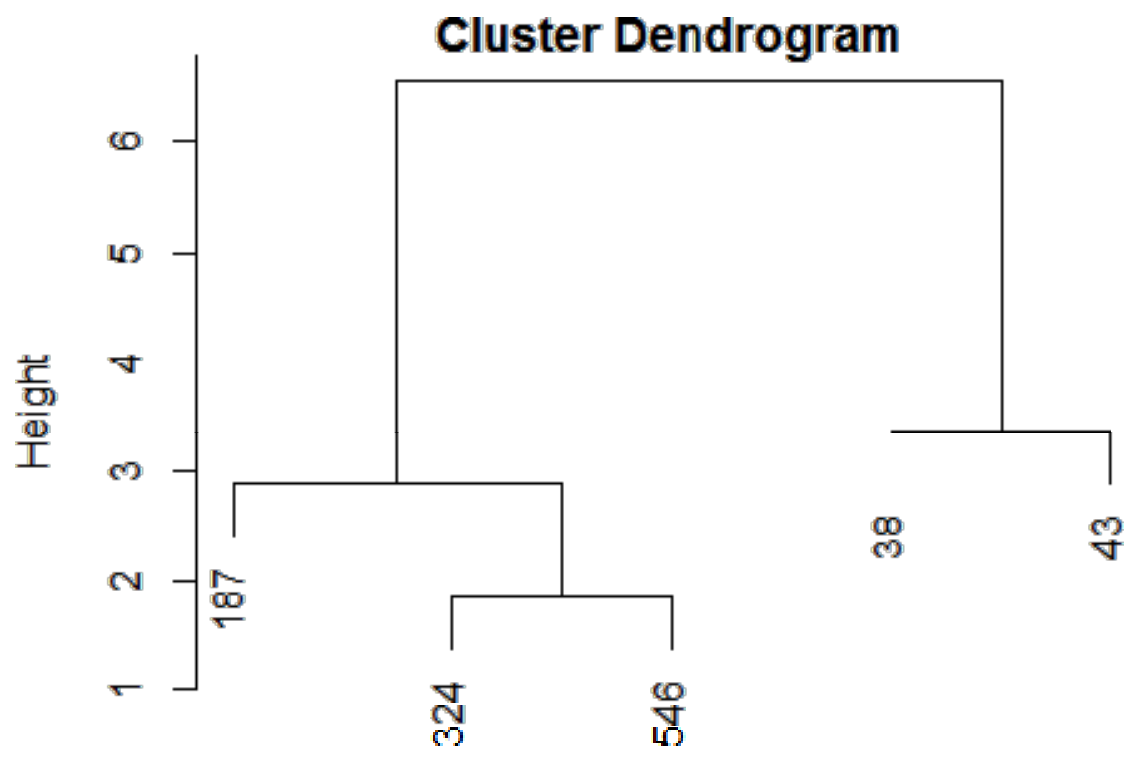

dist(data_scaled[clusters_Structures, ])

\#Build a heapmat of the crontribution of the achetypes to each sample \#Define plots margin parameters.

\#In case of "Error in plot.new() : figure margins too Large" resize the plot windows

\#in rstudio to be the full left panel.

$\operatorname{par}(\operatorname{mar}=c(1,1,1,1))$

heatmap.2(aa\$alphas[order $($ datasource [data_select_ID, color_ID] $)$, ], trace = "none", Rowv = F, density. info="none", dendrogram = "column",

Colv=as.dendrogram(hclust (dist (data_scaled[archetypal_structures, ]))), col=brewer . pal ( 7, "RdBu")) 


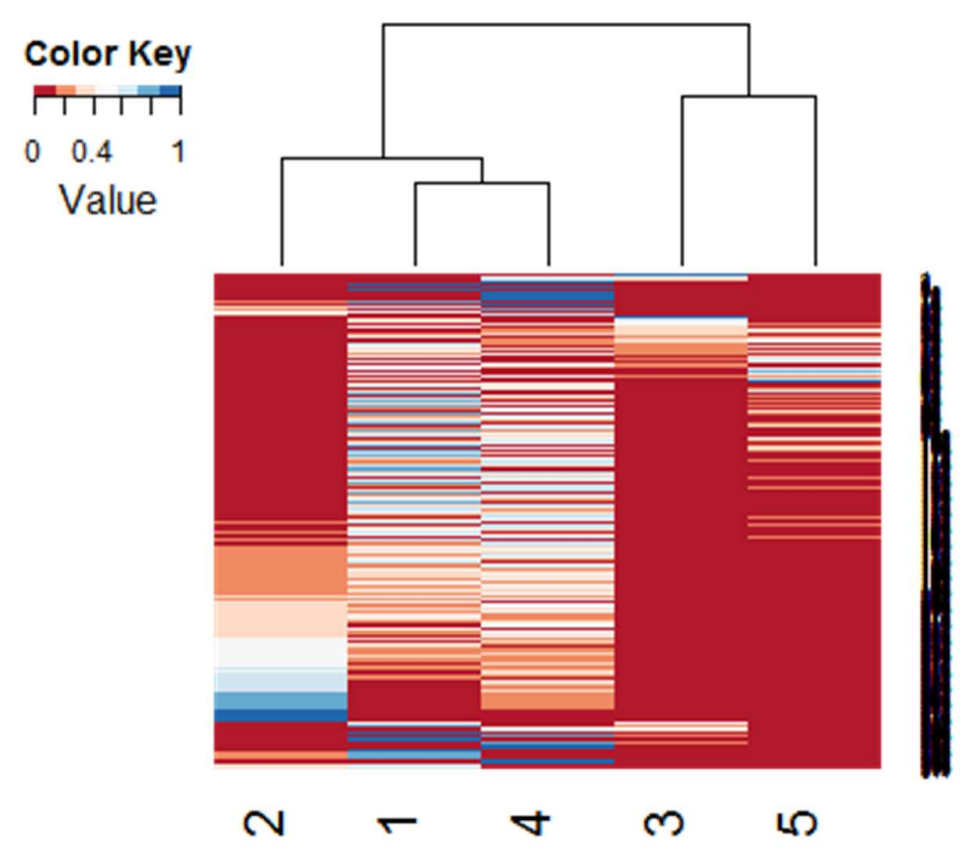

\#Build 3D scatter plots of the Principal Component Analysis (PCA) \#Archetype structures are bigger spheres and prototypes are shadowed spheres library (rgl)

rgl.open()

rgl.bg( sphere = FALSE, fogtype = "none", color=c("white","black"), back="lines")

for (i in 1:nrow $(p c a \$ x)$ )

\{rgl.spheres $(p c a \$ x[i, 1], p c a \$ x[i, 2], p c a \$ x[i, 3], 0.1$,

color $=c$ (as.numeric $($ datasource [data_select_ID $[i]$,

color_ID])))\}

rgl.spheres (pca $\$ \times[$ archetypal_Structures, 1], pca $\$ \times[$ archetypal_Structures, 2], pca $\$ \times[$ archetypal_Structures, 3],0.5,

color=c(as.numeric (datasource[data_select_ID[archetypal_Structures],

color_ID])))

rgl.spheres (pca\$x[clusters_Structures, 1], pca $\$ \times[$ clusters_Structures, 2], pca $\$ x[$ clusters_Structures, 3],0.5,

color $=c$ (as.numeric (datasource[data_select_ID[clusters_Structures],

color_ID])), alpha=0.5)

rgl.1ines $(c(\min (p c a \$ x[, 1])$ -

$1, \max (p c a \$ x[, 1])+1), c(\max (p c a \$ x[, 2])+1, \max (p c a \$ x[, 2])+1)$, $c(\min (\operatorname{pca} \$ x[, 3])-1, \min (\operatorname{pca} \$ x[, 3])-1), \operatorname{color}=c(" b l a c k "))$

$\operatorname{rgl.lines}(c(\min (p c a \$ x[, 1])-1, \min (p c a \$ x[, 1])-1), c(\min (p c a \$ x[, 2])-$ $1, \max (\operatorname{pca} \$ \times[, 2])+1)$, 


$$
c(\min (p c a \$ x[, 3])-1, \min (p c a \$ x[, 3])-1), \operatorname{color}=c(" b l a c k "))
$$

rgl.lines $(c(\min (p c a \$ x[, 1])-1, \min (p c a \$ x[, 1])-$

$1), c(\max (p c a \$ x[, 2])+1, \max (p c a \$ x[, 2])+1)$, $c(\min (\operatorname{pca} \$ x[, 3])-1, \max (\operatorname{pca} \$ x[, 3])+1)$, color $=c(" b l a c k "))$

rgl.viewpoint $(0,-90$, zoom $=0.7)$

rgl.texts $(\max (p c a \$ x[, 1])+1, \max (p c a \$ x[, 2])+1, \min (p c a \$ x[, 3])-2$, c("PC1") , cex=1.5, color=c ("black"), family=c("sans"))

$\operatorname{rgl.texts}(\min (\operatorname{pca} \$ \times[, 1])-1, \min (p c a \$ \times[, 2])+1, \min ($ pca $\$ \times[, 3])-2$, c("PC2"), cex=1.5, color=c("black"), family=c("sans"))

rgl.texts $(\min (p c a \$ x[, 1])-1, \max (p c a \$ x[, 2])+1, \max (p c a \$ x[, 3])+2$, c("PC3"), cex=1.5,

$$
\text { color }=c(\text { "black"), family=c("sans")) }
$$

\#Build simplex plots of the archetypes model

\#Archetype structures are bigger spheres and prototypes are shadowed spheres

angle $=-(90 / 180) * 3.14$

$M<-\operatorname{matrix}(c(\cos ($ angle $)$, -sin(angle $), \sin ($ angle $), \cos ($ angle $)), 2,2)$

rgl.open()

rgl.bg (sphere $=$ FALSE, fogtype $=$ "none", color=c("white", "black"), back="lines")

for ( $i$ in 1:length(archetypal_Structures) -1$)\{$

rgl.lines (c((sp \%*\% M) $[i, 1],($ sp \%*\% M) $[i+1,1]), c(($ sp \%*\% M) $[i, 2]$,

$$
c(\theta, \theta), \operatorname{color}=c(" b l a c k "))\}
$$

$(\mathrm{sp} \% * \% \mathrm{M})[i+1,2])$,

rgl.lines (c((sp \%*\% M) [length (archetypal_structures), 1$]$, (sp \%*\% $M)[1,1])$, c ((sp \%*\% M) [ length(archetypal_Structures), 2], (sp \%*\% M) [1,2]), $c(\theta, \theta)$, color $=c(" b l a c k "))$

rgl.spheres ((aa\$alphas\%*\%sp\%*\% M) [,1], (aa\$alphas\%*\%sp\%*\% M) [, 2], $r=0.3$, $\operatorname{rep}(\theta, \operatorname{nrow}($ data $))$, color $=$

as.numeric(datasource[data_select_ID, color_ID]))

rgl.spheres ((aa\$alphas\%*\%sp\%* M) [archetypal_Structures, 1], (aa\$alphas\%*\%sp\%*\% M) [archetypal_Structures, 2$], r=$

$0.7, \operatorname{rep}(\theta, \operatorname{nrow}($ data $))$, color $=$

as.numeric(datasource[data_select_ID,color_ID][archetypal_Structures]))

rgl.spheres ( (aa\$alphas\%*\%sp\%*\% M) [clusters_Structures, 1],

0.7, alpha=0.3, (aa\$alphas\%*\%sp\%*\% M) [clusters_Structures, 2 ], $r=$

$$
\operatorname{rep}(\theta, \operatorname{nrow}(\text { data })) \text {, }
$$
color $=$

as.numeric(datasource[data_select_ID, color_ID][archetypal_Structures]))

rgl.spheres (seq $(19,19+$

length (unique (datasource[data_select_ID,color_ID]))*(-0.), - $\theta_{\text {. }}$ ), $\operatorname{seq}(1,1+$

(length (unique (datasource[data_select_ID, color_ID])) -1)*0.6,0.6), $\operatorname{rep}(\theta$, length (unique (datasource[data_select_ID, color_ID])) )

,$r=0.3$,

color $=c$ (as.numeric (unique (datasource[data_select_ID, color_ID])) ))

rgl.texts (seq $(22.5,22.5+$ 
length(unique (datasource[data_select_ID, color_ID]))*(-0.), - 0. ), $\operatorname{seq}(1.05,1.05+$

(length (unique (datasource[data_select_ID, color_ID])) -1)*0.6,0.6), $\operatorname{rep}(\theta$, length (unique (datasource[data_select_ID, color_ID]))) ,

unique(datasource[data_select_ID, color_ID]), cex=1.5, color=c("black"), famil $y=c($ "sans"))

rgl.viewpoint $(0,0$, zoom $=0.7)$ 